\title{
Non-stationary axially symmetric goal of thermo-elasticity for a fixed rigid circular multi-layer plate
}

\author{
Dmitry Shlyakhin ${ }^{1 *}$ \\ ${ }^{1}$ Samara State Technical University, Institute of Architecture and Civil Engineering, 194, \\ Molodogvardeyskaya St., 443001, Samara, Russia
}

\begin{abstract}
This paper considers axially symmetric goal of thermoselasticity for a fixed rigid circular multi-layer isotropic plate with nonstationary heat flows on its front faces. The mathematical model is presented in the form of a not self-adjoint system that includes differential motion equations and a linear equation of the thermal-elastic state in a three-dimensional model. A new closed solution in an unconnected setting is built by the method of finite integral transformation. At the same time, a standardization procedure is conducted at each stage of the solution, which allows to implement an appropriate conversion algorithm. The calculated ratios provide an opportunity to perform a qualitative and quantitative analysis of the associated temperature and mechanical fields and also to examine stress-strain behaviour of the multi-layer systems both under the local thermal shock, which is observed, for example, in the interaction with the laser beam and in the case of uneven unsteady surface heating, which is frequent during the operation of protective construction structures.
\end{abstract}

\section{Introduction}

There are structures working under uneven heating which are used in construction, aviation and missile equipment as well as in a number of branches of mechanical engineering [1-6]. To describe their work, it is necessary to perform an in-depth analysis of non-stationary processes, without which it is impossible to understand the effect of temperature and mechanical stress fields. Typically, the design structure is presented as an infinitely long plate or an infinitely long cylinder. In other cases the task is investigated on the basis of the solution of the thermoelasticity equation only, without regard to the deformation of the building [3.4].

The task is greatly complicated when multi-layer finite dimension systems with a number of properties that qualitatively distinguish them from traditional structures are investigated [7-13]. In this case, the mathematical model of the initially boundary problem with account of the connection between movement and temperature, consists of a not self-adjoint system of differential equations of thermoelestic movement [14]. Their solution and fulfillment of boundary conditions is only possible for some elastic systems with some private

\footnotetext{
* Corresponding thor: d-612-mit2009@yandex.ru
} 
docking options [14-16]. In addition, the paper aims to try using accurate and approximative methods of calculation in their combination [4].

In this paper, in order to solve the non-stationary task of thermo-elasticity for a multilayer round rigid plate, the authors use methods of finite integral transformations (Hankel, Fourier, generic transformation) to obtain a closed solution in an unbound setting.

\section{Materials and methods}

Let a fixed rigid circular multi-layer plate in the cylindrical coordinate system occupies region $\left(r_{*}, \theta, z_{*}\right) \Omega:\left\{0 \leq r_{*} \leq b, 0 \leq \theta \leq 2 \pi, 0 \leq z_{*} \leq h^{*}\right\}$. For definiteness we assune that the construction consists of three layers. Besides, exterior and interior layers are in hard contact and have thickness $h_{1}^{*}$ and $h_{2}^{*}\left(h^{*}=2 h_{1}^{*}+h_{2}^{*}\right)$ respectively. Heat flows affect the end surfaces of the plate, $\omega_{1}^{*}\left(r_{*}, t_{*}\right), \omega_{2}^{*}\left(r_{*}, t_{*}\right)$ which are arbitrary time and radial coordinate functions $r_{*}$ and $t_{*}$.

Differential equations of motion and thermal conductivity in a linear position in the absence of internal heat sources, as well as the initially-boundary conditions in the dimensionless shape and in cylindrical coordinate system, are as follows [14]:

$$
\begin{aligned}
& \frac{\partial}{\partial r} \nabla U+\frac{\left(1-2 v_{j}\right)}{2\left(1-v_{j}\right)} \frac{\partial^{2} U}{\partial z^{2}}+\frac{1}{2\left(1-v_{j}\right)} \frac{\partial^{2} W}{\partial r \partial z}-B_{j} \frac{\partial T}{\partial r}-F_{j} \frac{\partial^{2} U}{\partial t^{2}}=0, \\
& \frac{\left(1-2 v_{j}\right)}{2\left(1-v_{j}\right)} \nabla \frac{\partial W}{\partial r}+\frac{\partial^{2} W}{\partial z^{2}}+\frac{1}{\left(1-v_{j}\right)} \frac{\partial}{\partial z} \nabla U-B_{j} \frac{\partial T}{\partial z}-F_{j} \frac{\partial^{2} W}{\partial t^{2}}=0, \\
& \nabla \frac{\partial T}{\partial r}+\frac{\partial^{2} T}{\partial z^{2}}-\frac{1}{k_{j}} \frac{\partial T}{\partial t}-\eta_{j} \frac{\partial}{\partial t}\left(\nabla U+\frac{\partial W}{\partial z}\right)=0 ; r=0,1 \\
& W(0, z, t)<\infty, \quad U(0, z, t)<\infty, T(0, z, t)<\infty, \\
& U(1, z, t)=0, W(1, z, t)=0, \quad \frac{\partial T}{\partial r}{ }_{\mid r=1}=0 \\
& z=0, h \quad \frac{\partial W}{\partial r}+\frac{\partial U}{\partial z}=0, \quad \nabla U+\frac{\left(1-v_{1}\right)}{v_{1}}\left(\frac{\partial W}{\partial z}+T\right)=0, \quad T=\omega_{1(2)} \\
& z=h_{1}, h_{1}+h_{2} \quad\left(\frac{\partial W}{\partial r}+\frac{\partial U}{\partial z}\right)_{\mid j=1}=b_{1}\left(\frac{\partial W}{\partial r}+\frac{\partial U}{\partial z}\right)_{\mid j=2}, \\
& {\left[\nabla U+\frac{\left(1-v_{1}\right)}{v_{1}}\left(\frac{\partial W}{\partial z}+T\right)\right]_{\mid j=1}=b_{2}\left[\nabla U+\frac{\left(1-v_{2}\right)}{v_{2}}\left(\frac{\partial W}{\partial z}+T\right)\right]_{\mid j=2},} \\
& U(z+0)=U(z-0), \quad W(z+0)=W(z-0), \quad T(z+0)=T(z-0) ;
\end{aligned}
$$




$$
\begin{aligned}
& t=0 \quad U(r, z, 0)=U_{0}(r, z), \dot{U}(r, z, 0)=\dot{U}_{0}(r, z), \\
& W(r, z, 0)=W_{0}(r, z), \dot{W}(r, z, 0)=\dot{W}_{0}(r, z), T(r, z, 0)=T_{0}(r, z) ; \\
& \text { where }\left\{U, W, r, z, h, h_{1}, h_{2}\right\}=\left\{U^{*}, W^{*}, r_{*}, z_{*}, h^{*}, h_{1}^{*}, h_{2}^{*}\right\} / b, \quad t=\frac{A}{b} t_{*} \\
& A=\sqrt{\frac{E_{1}\left(1-v_{1}\right)}{\left(1+v_{1}\right)\left(1-2 v_{1}\right) \rho_{1}}},\left\{T, \omega_{1}, \omega_{2}\right\}=\left\{T^{*}, \omega_{1}^{*}, \omega_{2}^{*}\right\} \frac{\left(1-v_{1}\right)}{\left(1+v_{1}\right) \alpha_{t}^{(1)}}, \quad B_{1}=1 \text {, } \\
& B_{2}=b_{3} \frac{\left(1+v_{2}\right)}{\left(1+v_{1}\right)} \frac{\alpha_{t}^{(2)}}{\alpha_{t}^{(1)}}, \quad F_{2}=\frac{b_{3}}{b_{1}} \frac{\rho_{2}}{\rho_{1}} \frac{\left(1-2 v_{2}\right)}{\left(1-2 v_{1}\right)}, \\
& F_{1}=1, \quad \eta_{j}=A E_{j} b \frac{\left(1-v_{1}\right)}{\left(1+v_{1}\right)\left(1-2 v_{j}\right)} \frac{\alpha_{t}^{(j)}}{\alpha_{t}^{(1)}} T_{0}, \quad k_{j}=\frac{k_{j}^{*}}{A b}, \nabla=\frac{\partial}{\partial r}+\frac{1}{r} \text {, } \\
& b_{1}=\frac{E_{2}\left(1+v_{1}\right)}{E_{1}\left(1+v_{2}\right)}, \quad b_{2}=b_{1} \frac{\left(1-2 v_{1}\right) v_{2}}{\left(1-2 v_{2}\right) v_{1}}, \quad b_{3}=\frac{\left(1-v_{1}\right)}{\left(1-v_{2}\right)},
\end{aligned}
$$

$U^{*}\left(r_{*}, z_{*}, t_{*}\right), W^{*}\left(r_{*}, z_{*}, t_{*}\right)$ - components of movement vector; $T^{*}-$ temperature change; $T_{0}$ - absolute temperature of the initial state of the body; $E_{j}, v_{j}, \alpha_{t}^{(j)}, k_{j}^{*}$ - elastic modulus, Poisson ratios, linear thermal expansion and thermal diffusivity of layer $j$.

The first three inequalities (2) are the regularity conditions for the solution of a continuous plate, and the latter equality takes into account lack of heat exchange between the plate and rigid fixing environment. Equations (4) are the conditions of joint stress, displacement, and temperature.

Formulae (1) - (5) represent a mathematical statement for the theory of thermoelasticity boundary problem.

Here, equalities (1) present a not self-adjoint system of equations for which a common integral cannot be obtained. It is therefore necessary to consider the taske (1) to (5) in an unbounded setting. New closed solutions of non-stationary tasks of thermoelasticity and elasticity theory are built by consistently using the method of partial separation of variables as finite integral transformations. The authors in consecutive stages use Hankel's transformations with finite limits along the axial coordinate and a generalized final transformation (kip) along the radial variable At the same time, a standardization procedure is conducted at each stage of the solution, which allows to implement an appropriate conversion algorithm.

\section{Results}

The first step is to solve the equation of thermoelasticity (1) relative to the function $T(r, z, t)$ with the corresponding initially boundary conditions:

$$
\begin{aligned}
& \nabla \frac{\partial T}{\partial r}+\frac{\partial^{2} T}{\partial z^{2}}-\frac{1}{k_{j}} \frac{\partial T}{\partial t}=\eta_{j} \frac{\partial}{\partial t}\left(\nabla U+\frac{\partial W}{\partial z}\right), \\
& r=0,1 \quad T(0, z, t)<\infty, \quad \frac{\partial T}{\partial r}{ }_{\mid r=1}=0 ;
\end{aligned}
$$




$$
\begin{gathered}
z=0, h \quad T(r, 0, t)=\omega_{1}(r, t) \quad T(r, h, t)=\omega_{2}(r, t) ; \\
z=h_{1}, h_{1}+h_{2} \quad T(z+0)=T(z-0), \\
{\left[\frac{\left(1-v_{1}\right)}{v_{1}} T\right]_{\mid j=1}-b_{2}\left[\frac{\left(1-v_{2}\right)}{v_{2}} T\right]_{\mid j=2}=b_{2}\left[\nabla U+\frac{\left(1-v_{2}\right)}{v_{2}} \frac{\partial W}{\partial z}\right]_{\mid j=2}-\left[\nabla U+\frac{\left(1-v_{1}\right)}{v_{1}} \frac{\partial W}{\partial z}\right]_{\mid j=1} ;} \\
t=0 \quad T(r, z, 0)=T_{0}(r, z) .
\end{gathered}
$$

It is initially assumed that functions $U, W$ are equal to zero. In this case we notice that the last condition (9) will not run.

Taking into account the theorems of solutions superposition and the linear nature of the original calculation ratios, we enter the new function $R(r, z, t)$ associated with the function $T(r, z, t)$, as follows:

$$
T=\left(r^{2}-2 r\right)\left(\omega_{1}+\omega_{2}\right)+R .
$$

This view allows the functions to be deferred to a $\omega_{1}, \omega_{2}$ series in the future if they do not change at the radial coordinate.

As a result of substitution (11) in (6) - (10), a new task of thermoelasticity is obtained in relation to function $R$. This task is solved by the method of Hankel's final integral transformations along radial coordinate using the following transformant and the reciprocation formula:

$$
R_{H}(n, z, t)=\int_{0}^{1} R(r, z, t) r J_{0}\left(j_{n} r\right) d r, \quad R(r, z, t)=2 \sum_{n=0}^{\infty} \frac{R_{H}(z, t) J_{0}\left(j_{n} r\right) d r}{J_{0}\left(j_{n}\right)^{2}} .
$$

Where $j_{n}-$ is positive zeros of function $J_{1}\left(j_{n}\right)\left(n=0, j_{0}=0\right), J_{0}(\ldots), J_{1}(\ldots)-$ the Bessel function of the first type of zero and first order.

When resolving a task relative to transformant $R_{H}$, we use a generalized final integral transformation (kip) [18.19] is used along the radial variable:

$$
\begin{gathered}
L_{H}(n, m, t)=\int_{0}^{h} R_{H}(n, z, t) N\left(\lambda_{m}, z\right) d z, \\
R_{H}(n, z, t)=\sum_{m=0}^{\infty} L_{H}(n, m, t) N_{1}\left(\lambda_{i m}, z\right)\left\|N_{i m}\right\|^{2},\left\|N_{i m}\right\|^{2}=\int_{0}^{h} N_{1}\left(\lambda_{i m}, z\right)^{2} d z .
\end{gathered}
$$

Where $N_{1}\left(\lambda_{\text {im }}, z\right)$ is a function of transformation edge along function $z, \lambda_{\text {im }}-$ - its own values makeing up the counting set. 
Applying reciprocation formula (12), (13) in consecutive stages to the transformant $L_{H}(n, m, t)$, with account of (11), we obtain the expression for $T(r, z, t)$ :

$$
T=\left(r^{2}-2 r\right)\left(\omega_{1}+\omega_{2}\right)+\frac{4}{h} \sum_{j=0}^{\infty} \frac{J_{0}\left(j_{n} r\right)}{J_{0}\left(j_{n}\right)^{2}} \sum_{m=1}^{\infty} L_{H}(n, m, t) N\left(\lambda_{m}, z\right),
$$

The second step is to solve a tsk of the theory of elasticity with regard to function $U, W$ based on a given (well-known) function $T$ :

$$
\begin{aligned}
& \frac{\partial}{\partial r} \nabla U+\frac{\left(1-2 v_{j}\right)}{2\left(1-v_{j}\right)} \frac{\partial^{2} U}{\partial z^{2}}+\frac{1}{2\left(1-v_{j}\right)} \frac{\partial^{2} W}{\partial r \partial z}-F_{j} \frac{\partial^{2} U}{\partial t^{2}}=B_{j} \frac{\partial T}{\partial r}, \\
& \frac{\left(1-2 v_{j}\right)}{2\left(1-v_{j}\right)} \nabla \frac{\partial W}{\partial r}+\frac{\partial^{2} W}{\partial z^{2}}+\frac{1}{\left(1-v_{j}\right)} \frac{\partial}{\partial z} \nabla U-F_{j} \frac{\partial^{2} W}{\partial t^{2}}=B_{j} \frac{\partial T}{\partial z} \\
& r=0,1 \quad W(0, z, t)<\infty, \quad U(0, z, t)<\infty, \quad U(1, z, t)=0, \quad W(1, z, t)=0 ; \\
& z=0, h \quad \frac{\partial W}{\partial r}+\frac{\partial U}{\partial z}=0, \quad \nabla U+\frac{\left(1-v_{1}\right)}{v_{1}} \frac{\partial W}{\partial z}=-\frac{\left(1-v_{1}\right)}{v_{1}} T \\
& z=h_{1}, h_{1}+h_{2} \quad\left(\frac{\partial W}{\partial r}+\frac{\partial U}{\partial z}\right)_{\mid j=1}=b_{1}\left(\frac{\partial W}{\partial r}+\frac{\partial U}{\partial z}\right)_{\mid j=2}, \\
& {\left[\nabla U+\frac{\left(1-v_{1}\right)}{v_{1}} \frac{\partial W}{\partial z}\right]_{\mid j=1}-b_{2}\left[\nabla U+\frac{\left(1-v_{2}\right)}{v_{2}} \frac{\partial W}{\partial z}\right]_{\mid j=2}=b_{2}\left[\frac{\left(1-v_{2}\right)}{v_{2}} T\right]_{\mid j=2}-\left[\frac{\left(1-v_{1}\right)}{v_{1}} T\right]_{\mid j=1},} \\
& U(z+0)=U(z-0), \quad W(z+0)=W(z-0) ; \\
& t=0 \quad U(r, z, 0)=U_{0}(r, z), \dot{U}(r, z, 0)=\dot{U}_{0}(r, z), \\
& W(r, z, 0)=W_{0}(r, z), \dot{W}(r, z, 0)=\dot{W}_{0}(r, z) .
\end{aligned}
$$

To accomplish tasks (15) - (19), Hankel's finite integral transformations along the radial coordinate and the generalized transformation (kip) [18.19] are used on the axial variable. Firstly, formulae (15) - (19) are reduced to a standard form making it possibly to carry put a procedure of separation of variables along radial coordinate. For that purpose, Formula 12 is changed for that of absence of shearing stress:

$$
\sigma_{r z \mid r=1}=\frac{C_{55}^{(s)}}{C_{11}^{(s)}}\left(\frac{\partial W}{\partial r}+\frac{\partial U}{\partial z}\right)=0
$$

and on the basis of the superposition of solution theorem, a new function $w(r, z, t)$ related to ratio $W(r, z, t)$ is introduced:

$$
W(r, z, t)=W_{1}(t)+w(r, z, t)
$$


where $W_{1}(t)$ - is twice-differentiated function that is defined in the process of resolving the task from the condition that there are no vertical movements of the median surface of the plate under $r=1$.

When we put (21) in (15) - (19), (20), we obtain a new boundary problem as regard to functions $U, w$. Here, the boundary conditions of the elasticity theory (16) take the form:

$$
r=0,1 \quad U(1, z, t)=0, \quad \frac{\partial w}{\partial r}{ }_{\mid r=1}=0, \quad U(0, z, t)<\infty, w(0, z, t)<\infty .
$$

We a apply Hankel transformation with the finite bound along variable $r$ to the boundary problem $U, w$ using the following transformants and reciprocation formula:

$$
\begin{gathered}
u_{H}\left(j_{n}, z, t\right)=\int_{0}^{1} U(r, z, t) r J_{1}\left(j_{n} r\right) d r, \quad w_{H}\left(j_{n}, z, t\right)=\int_{0}^{1} w(r, z, t) r J_{0}\left(j_{n} r\right) d r, \\
U(r, z, t)=2 \sum_{n=1}^{\infty} \frac{u_{H}(n, z, t)}{J_{0}\left(j_{n}\right)^{2}} J_{1}\left(j_{n} r\right), \quad w(r, z, t)=2 \sum_{n=0}^{\infty} \frac{w_{H}(n, z, t)}{J_{0}\left(j_{n}\right)^{2}} J_{0}\left(j_{n} r\right) .
\end{gathered}
$$

Using transformations (23) allows us to obtain the boundary problem relative to the functions $u_{H}, w_{H}$. To further implement the solution algorithm, it is necessary to adopt heterogeneous boundary conditions for the variable $z$ to homogeneou conditions. To do this, we introduce a new function $W_{H}$ related to $w_{H}$ by the ratio:

$$
w_{H}(n, z, t)=H_{1}(n, z, t)+W_{H}(n, z, t) .
$$

Where $H_{1}(n, z, t)-$ is the function that allows the conversion of boundary conditions.

We solve the obtained initially boundary task for $u_{H}, W_{H}$ by using a structural algorithm of the KIP method, entering $[0, h]$ instrument with unknown components on the segment $K_{1}\left(\lambda_{i n} r\right), K_{2}\left(\lambda_{i n} r\right)$ :

$$
\begin{gathered}
G\left(\lambda_{i n}, n, t\right)=\int_{0}^{h}\left[u_{H}(n, z, t) K_{1}\left(\lambda_{i n}, z\right)+W_{H}(n, z, t) K_{2}\left(\lambda_{i n}, z\right)\right] d z, \\
u_{H}(n, z, t)=\sum_{i=1}^{\infty} G\left(\lambda_{i n}, n, t\right) K_{1}\left(\lambda_{i n}, z\right)\left\|K_{i n}\right\|^{-2}, \\
W_{H}(n, z, t)=\sum_{i=1}^{\infty} G\left(\lambda_{i n}, n, t\right) K_{2}\left(\lambda_{i n}, z\right)\left\|K_{i n}\right\|^{-2}, \\
\left\|K_{i n}\right\|^{2}=\int_{0}^{h}\left[K_{1}^{2}\left(\lambda_{i n}, z\right)+K_{2}^{2}\left(\lambda_{i n}, z\right)\right] d z .
\end{gathered}
$$

The use of the KIP method results in expressions for the component $K_{1}\left(\lambda_{i n} r\right), K_{2}\left(\lambda_{i n} r\right)$ and transformant $G\left(\lambda_{i n}, n, t\right)$. Entering $K_{1}\left(\lambda_{i n} r\right), K_{2}\left(\lambda_{i n} r\right)$ in a variable $z$ boundary conditions 
creates a system of algebraic equations that allow to define permanent integrations $D_{\text {lin }} . . D_{12 \text { in }}$ and own values $\lambda_{\text {in }}$.

The final expression of functions $U(r, z, t), W(r, z, t)$ are obtained by applying to transformant $G\left(\lambda_{i n}, n, t\right)$ reciprocation formula KIP (25) and Hankel's final transformations (23) in consecutive stages. As a result, taking into account (21), (24), we have:

$$
\begin{gathered}
U(r, z, t)=2 \sum_{n=1}^{\infty} \frac{J_{1}\left(j_{n} r\right)}{J_{0}\left(j_{n}\right)^{2}}\left[\sum_{i=1}^{\infty} G\left(\lambda_{i n}, n, t\right) K_{1}\left(\lambda_{i n}, z\right)\left\|K_{i n}\right\|^{-2}\right], \\
W(r, z, t)=W_{1}(t)+2 \sum_{n=0}^{\infty} \frac{J_{0}\left(j_{n} r\right)}{J_{0}\left(j_{n}\right)^{2}}\left[H_{1}(n, z, t)+\sum_{i=1}^{\infty} G\left(\lambda_{i n}, n, t\right) K_{2}\left(\lambda_{i n}, z\right)\left\|K_{i n}\right\|^{-2}\right] .
\end{gathered}
$$

Function $W_{1}(t)$ can be found on condition when the middle surface is stationary with account of $r=1$.

$$
W_{1}(t)=-2 \sum_{n=0}^{\infty} J_{0}\left(j_{n}\right)^{-1} \sum_{i=1}^{\infty} G\left(\lambda_{i n}, n, t\right) K_{2}\left(\lambda_{i n}, h / 2\right)\left\|K_{i n}\right\|^{-2}
$$

The resulting calculation ratio (26) satisfies the differential equations (15) and boundary conditions (16) - (19) (the last boundary condition (16) is only performed for the medial surface of the plate), i.e. represent a closed solution of the elasticity theory.

\section{Discussion}

The calculated functions $U, W$ in the next phase of the research allow to improve value $T$. This iterative process is performed until the numerical results of the boundary tasks in question are matched with the specified precision.

For the purpose of the elasticity theory, the primary assumption is to replace the last boundary condition (16) by (20). As a result, the cylindrical surface of the structure is docked with the additional function $W_{1}(t)$ used only at one point at the height of the section. Paper [13] shows that in case of a bi-morph plate, this assumption results in a slight change in the tense and deformed state and can be used in the study of thin multi-layer structures.

It is also important that in the present paper, when considering an axisymmetric initially-boundary problem of thermoelasticity has theory, the authors took into account the effect of the effect of reinforcement of a multi-layer structure on its dynamic reaction in the case of operation under nonstationary heat flows and to build a closed solution as a $3 \mathrm{~d}$ production.

\section{Conclusions}

On the basis of our algorithm the following conclusions can be formulated:

1) A new closed solution has been built within the framework of the thermoelasticity theory for a three-layer rigidly fixed circular plate affected by temperature effect on its front faces. This approach makes it possible to abandon various kinematic hypotheses used in applied theories. 
2) The introduced algorithm also works for composite plates with an arbitrary number of layers and with different physical and elastic properties, which are placed symmetrical and asymmetrical in relation to the middle surface.

3) The temperature asymmetrical load, which is applied to the end surfaces of the structure as defined in general terms, allows the study of broad range of tasks of thermoelasticity.

4) The achieved ratio design makes it possible to determine not only the stress-strain behaviour of the system in question, but also the thickness of the layers and physical characteristics of the material necesary for the effective work of the construction.

5) The calculated ratios allow to determine the frequency spectrum of multi-layer structure own variations without regard of dissipative forces.

\section{References}

1. Yu.S. Vytchikov, I.G. Belikov, E.N. Mokhrina, Urban Construction and Architecture 3, 103-110 (2014) DOI:10.17673/Vestnik. 2014.03.18.

2. Yu.S. Vytchikov, M.E. Saparev, Urban Construction and Architecture 4, 108-114 (2015) DOI:10.17673/Vestnik.2015.04.15.

3. V.A. Kudinov, A.V. Eremin, A.E. Kuznetsova, E.V. Stefanuk, Higher education establishments news. Aviation Equipment 1, 30-35 (2014)

3. V.A. Kudinov, A.E. Kuznetsova, A.V. Eremin, E.V. Kotova, Vestnik of Samara State Technical University Series: Physico-Mathematical Sciences 1 (30), 215-221 (2013)

5. T.A. Golova, A.P. Denisova, Civil Engineering magazine 8, 9-19 (2014)

6. M.E. Frantsev, Shipbuilding 1, 38-42 (2005)

7. I.S. Makarova, Vestnik of Samara State Aerospace University 5 (36), 108-111 (2012)

8. D.C.D. Oguamanam, J.S. Hansen, G.R. Heppler, Journal Applied Mechanics 1(71), 49$56(2004)$

9. N.V. Smetankina, E.V. Svet, A. Shupikov, Problems of Mechanical Engineering 3 (7), 25-31 (2004)

10. E.I. Grigoluk, G.M. Kulikov, Vestnik TGU 2a(11), 439-448 (2005)

11. G. I. Rastorguev, S.I. Snisarenko, Applied Mechanics and Technical Physics 1 (50), 187-196 (2009)

12. Yu.E. Senitskiy, I.E. Kozma, Vestnik of Samara State Technical University Series: Physico-Mathematical Sciences 43, 52-67 (2006)

13. D. Shlyakhin, Procedia Engineering 150, 68-73(2016). DOI: 10.1016/j. proeng. 2016.06.717

14. S.A. Lychev, Vestnik of Samara State Technical University Natural Studies Series 4 (30), 118-124 (2003)

15. S.A. Lychev, A.V. Manzhirov, S.V. Uber, Bullutin of Russian Academy of Sciences Solid Mechanics 4, 138-154 (2010)

16. S.S. Kurennov, A. G. Nikolaev, Applied Mechanics and Technical Physics 1 (45), 9298 (2004)

17. P.N. Knyazev, Integral transformations (Moscow, Editorial URSS, 2004)

18. Yu.E. Senitskiy, News of Saratov University. New series. Series: Mathematics. Mechanics. Informatics 3-1 (11), 61-89 (2011)

19. Yu. E. Senitskiy, Procedia Engineering 153, 649-654 (2016)

20. D.A. Shlyahin, Industrial and civil engineering 6, 11-15 (2015) 